\title{
Electrochemical Hydrogen Pumping using a Platinum Catalyst made in a Fluidized Bed via Atomic Layer Deposition
}

\author{
A. M. Lubers ${ }^{1}$, A. W. Drake ${ }^{1}$, D. J. Ludlow ${ }^{2}$, \& A. W. Weimer ${ }^{1}$, \\ (1) Department of Chemical and Biological Engineering, University of Colorado Boulder, Boulder, \\ Colorado 80309 \\ (2) Center for Automation Systems and Technologies, Rensselaer Polytechnic Institute, Troy, New York \\ 12180 \\ *corresponding author email: alan.weimer@colorado.edu, phone: 303-492-3759
}




\begin{abstract}
:
Platinum nanoparticle catalysts for electrochemical hydrogen pumping were synthesized on a functionalized powder carbon substrate (XC72R) using atomic layer deposition (ALD) in a fluidized bed reactor (FBR). Trimethyl(methylcyclopentadienyl)platinum(IV) $\left(\mathrm{MeCpPtMe}_{3}\right)$ was used as the reagent for platinum delivery. Following deposition, $\mathrm{McCpPtMe}_{3}$ ligands were combusted or hydrogenated to yield platinum on the XC72R surface. Reactions throughout the ALD cycle were monitored using mass spectrometry and IR spectroscopy to clarify the deposition chemistry. The resultant platinum catalysts were compared to commercial products in hydrogen pumping tests. Hydrogenation made finer, more dispersed, platinum nanoparticles that performed similarly to their commercial equivalent when pumping hydrogen. Conversely, oxygenation made a coarser catalyst that underperformed its commercial equivalent. Thus, altering chemistries show potential for improving ALD catalyst performance.
\end{abstract}

\title{
Keywords:
}

Platinum, catalysts, hydrogen pumping, fluidized bed, fluidization, atomic layer deposition

\subsection{Introduction:}

Platinum catalysts are integral components of many chemical processes including catalytic reforming, catalytic conversion, and electrochemical power generation. As an expensive system constituent, optimal platinum use is desired, especially in emerging technologies. The use of platinum in electrochemical hydrogen pumping is one such application. Given the rising hydrogen economy [1], hydrogen separation from other gases such as $\mathrm{CO}$ and $\mathrm{CO}_{2}$ will become increasingly important [2]. Electrochemical pumps have been utilized to purify and pressurize hydrogen for several decades [3]. The process can be tailored to reaction conditions by choice of ion conductor [4] and is readily integrated into larger chemical systems [5]. Just as with a hydrogen fuel cell, these pumps catalytically split hydrogen into protons and electrons at the anode. Protons conduct through the membrane, then catalytically combine with electrons from the circuit to yield a hydrogen permeate, free of gas impurities, at the cathode (similar to water electrolysis). 
Electric power is consumed, not produced (Figure 1). Platinum is the most effective catalyst when coupled with proton exchange membranes $[6,7]$. In these devices, low loadings of platinum nanoparticles are needed to maximize the number of reaction sites while minimizing cost [8]. Atomic layer deposition (ALD) is suited to the production of these optimal materials.

ALD is a versatile engineering tool for precisely depositing metals, oxides, and polymers on particles [911]. Using volatile precursors, materials of choice can be deposited over the entire surface of a substrate particle [12]. A second precursor removes any unwanted ligands or deposits more organics, growing an array of materials one sub-monolayer at a time. ALD can be implemented on powder substrates in fluidized beds [13-16]. The fluidized bed allows nearly $100 \%$ efficient precursor utilization, high throughput, and straightforward scale-up [17, 18]. Practical applications include passivating photoactive particles [19], fabricating supported catalysts [20], and coatings for energy materials [21].

When platinum is deposited via ALD, nanoparticles are produced on most substrates, especially on particulate carbon [22-25] through an island growth mechanism [26]. In electrochemical contexts, the electrical conductivity of carbon is beneficial, although this material is poorly functionalized relative to alternative substrates [27]. This deficiency can be overcome through the generation of functional sites by carbon pretreatment. Functionalization renders ALD reactions more controllable [27]. Compared to incipient wetness and sputtering, ALD is a better method for precisely depositing nanosized catalysts on supports [28]. The ability to exchange precursors for inexpensive non-platinum materials is an advantage in producing future catalysts using ALD [29, 30].

This report is the first demonstration of platinum ALD on a carbon powder substrate for electrochemical hydrogen pumping. In brief, XC72R carbon was functionalized and used as a substrate for platinum ALD. Platinum nanoparticles were deposited using trimethyl(methylcyclopentadienyl)platinum(IV) $\left(\mathrm{MeCpPtMe}_{3}\right)$ as the first precursor and either oxygen or hydrogen as a second precursor. The resulting 
catalyst was integrated into electrodes, evaluated via hydrogen pumping, and compared to a commercial $\mathrm{Pt} / \mathrm{C}$ catalyst of equivalent weight loading. The process from carbon support selection to hydrogen pumping is summarized in Figure 2. Although the commercial catalyst initially outperformed the ALD catalyst using oxidation chemistry, a switch to a hydrogenation chemistry synthesis produced a competitive ALD catalyst.

\subsection{Materials \& Methods:}

\subsection{Functionalizing carbon}

Ten grams of Vulcan XC72R carbon from Cabot Corporation were modified by refluxing in $150 \mathrm{~mL}$ of $15 \mathrm{M}$ concentrated nitric acid at $120^{\circ} \mathrm{C}$ for 9 hours. The carbon was filtered, and then rinsed four times with DI water. The filtered modified carbon was dried in a cake overnight at room temperature to release absorbed water, and then was ground to a fine powder with a mortar and pestle. The surface groups on the functionalized carbon were characterized using diffuse reflectance infrared Fourier transform spectroscopy (DRIFTS), obtained using a Thermo Scientific Nicolet 6700 FT-IR with a closed cell attachment (Harrick). DRIFTS spectra were taken against a background of unmodified XC72R. The change in surface area owing to nitric acid treatment and platinum deposition was monitored using Brunauer, Emmett and Teller (BET) analysis measured by a Micrometics Gemini V surface area and pore size analyzer. Analyzed samples were prepared by evacuating at $100{ }^{\circ} \mathrm{C}$ for over 8 hours using a Micrometics VacPrep 061 sample degas system. Change in monatomic nitrogen and oxygen content was monitored using a TC600 from LECO Corporation.

\subsection{Pt ALD in a fluidized bed}

To deposit platinum [31], we placed 2 grams of the functionalized XC72R in the fluidized bed reactor. See Figure 3 for a schematic of an ALD FBR system. The ALD reactor was evacuated to 300 mtorr and the substrate dried overnight. The reactor sustained a temperature of $300{ }^{\circ} \mathrm{C}$ for ALD reactions with supporting line temperatures set at $115^{\circ} \mathrm{C}$ to facilitate efficient precursor flow through the ALD reactor system, under

vacuum conditions between 300 and 3000 mtorr. A bubbler containing 
trimethyl(methylcyclopentadienyl)platinum(IV) $\left(\mathrm{MeCpPtMe}_{3}\right)$ was heated to $40{ }^{\circ} \mathrm{C}$ to maintain a constant vapor pressure. $\mathrm{MeCpPtMe}_{3}$ was dosed by flowing $5 \mathrm{sccm}$ of nitrogen through the bubbler to entrain the organometallic precursor, for a dose time of 40 minutes. Pure nitrogen was used as a purge gas for 70 minutes at $5 \mathrm{sccm}$. Pure oxygen or $20 \%$ hydrogen balance argon as a second precursor was dosed at $5 \mathrm{sccm}$ for 90 minutes. To finish the cycle, pure nitrogen was purged through the system for 180 minutes at $5 \mathrm{sccm}$. One cycle was 380 minutes total. Five total cycles of platinum ALD were performed for each ALD catalyst material. An in-line Stanford Research Systems high pressure gas analyzer series QMS200 mass spectrometer was used to monitor by-products of the ALD reactions. Periodic spectra measuring partial pressure of species between 1 and 200 atomic mass units (AMU) were recorded. Spectra from each time point of relevant tracked species were compiled into pressure vs time graphs. The deposited platinum particles were analyzed for weight loading using inductively coupled plasma optical emission spectroscopy after digestion in an HF/aqua regia solution for 24 hours. Images of the platinum particles were produced using transmission electron microscopy (TEM) on a Philips CM-100 microscope. Platinum particle characteristics, including size, dispersion, and metal surface area, were measured by hydrogen uptake at 40 ${ }^{\circ} \mathrm{C}$ using a Quantachrome AS-1 Autosorb.

\subsection{Pt ALD in a vacuum chamber}

Separately, to inspect the surface groups during the ALD process using oxygen as a second precursor, platinum was deposited on the functionalized carbon while monitored by IR spectroscopy in a vacuum chamber. Since this experimental technique was only for analysis, no actual catalyst was produced. Functionalized XC72R was prepared for analysis by diluting $50 \%$ with $\mathrm{KBr}$. The diluted powder was pressed into a tungsten grid and fixed in a sample holder. The holder was placed in a vacuum chamber with windows for transmittance IR spectroscopy, performed by a Nicolet 6700 FTIR spectrometer. A nitrogen jacket around the chamber helped minimize noise generated from atmospheric gases. The powder in the holder was resistively heated to $300{ }^{\circ} \mathrm{C}$. Dosing $\mathrm{MeCptPtMe}_{3}$ consisted of isolating the chamber from vacuum and exposing it to a section containing precursor heated to $37{ }^{\circ} \mathrm{C}$ for 15 minutes. The precursor 
section was then isolated and the chamber was evacuated to simulate a purge for 10 minutes. Pure oxygen was dosed into the chamber as the second precursor step for 10 minutes followed by 20 minutes of vacuum evacuation for a subsequent purge. At various time points, IR spectra were recorded to inspect the species on the surface of the carbon powder throughout the ALD cycle. A total of two ALD cycles were performed using this analysis technique.

\subsection{Electrochemical hydrogen pumping}

Details of each type of membrane electrode assembly (MEA) evaluated for hydrogen pumping are summarized in Table 1. Due to the difference in cathode material, each ALD catalyst was compared to a commercial catalyst with similar weight loading on the anode. In order to reduce the platinum weight loading but maintain carbon loading for the catalyst layer, $\mathrm{Pt} / \mathrm{C}$ catalysts were mixed with excess $\mathrm{XC} 72 \mathrm{R}$ before ink synthesis. The resulting mixture was fabricated into an ink of the following composition: $3 \%$ $\mathrm{Pt} / \mathrm{C}$ mixture, $10 \%$ water, 22\% Nafion dispersion (LQ-1105 - $1100 \mathrm{EW}$ at 5\% weight), and 65\% methanol. The ink was shaken, then sonicated for 4 hours, and sprayed onto a Sigracet GDL 25 BC gas diffusion layer using a Harder \& Steenbeck Evolution airbrush to form the gas diffusion electrode (GDE). GDEs were left to dry overnight at room temperature. GDEs for the anode and cathode were bonded to wet proton conduction membranes (Nafion 212) for 5 minutes at $130{ }^{\circ} \mathrm{C}$ and 750 psi to produce membrane electrode assemblies (MEAs). MEAs were placed in single cell hardware with an active area of $10 \mathrm{~cm}^{2}$. At atmospheric pressure, cell temperature was maintained at $60{ }^{\circ} \mathrm{C}$, gas lines at $80{ }^{\circ} \mathrm{C}$, and gas flows were humidified with a dew point of $55^{\circ} \mathrm{C}, 78 \%$ relative humidity. Pure hydrogen was fed at a 1.2 stoichiometric rate from a Ceres Technologies PEM 221. The cathode was initially purged with pure nitrogen for 2 minutes. After hydrogen pumping at about 1 amp for 1 minute, nitrogen flow was stopped and the cathode gas inlet was plugged, allowing pumped hydrogen gas to flood the cathode and vent to atmospheric pressure. No sweep gas was used on the cathode while pumping. Voltage applied to the cell for hydrogen pumping was supplied from a Sorensen DCR 20-80B power supply. 


\subsection{Results and Discussion:}

This section will describe the modification of carbon (3.1), the chemistry of ALD using oxygen as a second precursor (3.2), the hydrogen pumping performance of the ALD catalyst produced using oxygen as a second precursor (3.3), the chemistry of ALD using hydrogen as a second precursor (3.4), and the hydrogen pumping performance of the ALD catalyst produced using hydrogen as a second precursor (3.5).

\subsection{Functionalization of XC72R carbon}

Atomic layer deposition reactions can be facilitated by surface oxides, which provide nucleation opportunities [32]. Since carbon is largely inert and does not naturally contain non-carbonaceous surface species, functionalization is beneficial. The application of acids to carbon is known to oxidize and produce oxygen-containing groups on the surface [33]. Detected anhydride and carbonate surface groups formed on the XC72R due to covalent functionalization by nitric acid are shown in Figure 4. Evidence of additional monatomic oxygen as well as nitrogen can be seen in Figure 5, likely added during the acid treatment. $\mathrm{MeCpPtMe}_{3}$ should attach more readily to these surface groups than the carbon support. A consequence of the functionalization process is the removal of carbon from the surface, which reduces the substrate surface area as seen in Figure 5.

\subsection{Pt ALD using oxygen as a second precursor in a fluidized bed and vacuum chamber}

The platinum nanoparticles produced by ALD using oxygen as a second precursor are shown in Figure 6. The platinum particle size, weight loading on carbon, and dispersion are compared to a commercial catalyst, HiSPEC 4000. The long dose times and many cycles executed contribute to the high weight loading of the platinum deposited on the carbon. To better understand platinum ALD using oxygen as a second precursor and to forecast platinum ALD scale-up we utilized in-line mass spectrometry to inspect reaction byproducts [17]. The pressures of gaseous species are plotted against time as shown in Figure 7 in order to track their evolution during each step of the second ALD cycle. 
Species related to combustion during an ALD cycle using the fluidized bed are shown in Figure 7, Part A. During the platinum dose, where $\mathrm{MeCpPtMe}_{3}$ deposits on the carbonaceous surface, an increase in $\mathrm{CO}_{2}$ is observed. This increase most likely results from $\mathrm{MeCpPtMe}_{3}$ reacting with surface functional groups and combining with adsorbed oxygen. A second $\mathrm{CO}_{2}$ spike is seen with the onset of oxygen dosing. Oxygen is used to burn away the platinum ligands, but also likely erodes the carbon surface and contributes to surface area contraction of the substrate. Water generation was not apparent, perhaps because the partial pressure was consistently high and, therefore, could mask subtle changes. The deduced combustion mechanisms for Pt ALD on carbon appear similar to those found on oxides [28, 34].

An increase in hydrocarbon species appears during the platinum dose as shown in Part B of Figure 7. Hydrocarbons likely attach to the surface during the self-catalytic decomposition of $\mathrm{MeCpPtMe}_{3}$ upon reaction with groups on the substrate surface and adsorbed oxygen, a phenomenon explored by Mackus et al [35]. Butane generation was not observed, cyclopentene partial pressure scaled with propane and MeCp, and hydrogen scaled with methane and ethane. Hydrocarbon signals during the $\mathrm{N}_{2}$ purge and $\mathrm{O}_{2}$ dose likely owe to the lingering recombination and desorption of platinum ligands, alongside any catalytic action of platinum on the carbon surface.

To further understand the reactions that occur, Pt ALD using oxygen as a second precursor was also performed in a vacuum chamber where the surface of the carbon was analyzed with in situ IR spectroscopy. Spectra from each step of the second ALD cycle are shown in Figure 8. Before ALD is performed, the peak at $1370 \mathrm{~cm}^{-1}$ relates to the peak in Figure 4 for anhydride functional groups. With each subsequent part of the dose, this peak dissipates, likely due to combustion of surface hydrocarbon groups, either being consumed by reaction, or being covered by deposited combustion product material. The peaks spanning from 2300 to $2380 \mathrm{~cm}^{-1}$ correspond to carbon dioxide formation in the chamber [36]. The $\mathrm{CO}_{2}$ peaks are 
present for both the platinum dose and oxygen dose, which support the $\mathrm{CO}_{2}$ generation shown in the mass spectra in Part A of Figure 7.

\subsection{Hydrogen pumping with Pt catalysts from ALD using oxygen as a second precursor}

The catalyst synthesized via ALD using oxygen as a second precursor was compared to HiSPEC 4000 for hydrogen pumping ability, results are summarized in Figure 9. The impedance to ion transport typically dominates electrochemical hydrogen pumping voltages, leading to very linear voltage versus current behavior (as demonstrated with HiSPEC 4000, Figure 9). The larger voltages observed at higher currents arise from resistance to reactant diffusion and charge transfer kinetics [37]. Given that the MEAs were all prepared by the same protocol, it was assumed that pumping differences were largely attributable to the catalyst.

The commercial catalyst (HiSPEC 4000) showed the best performance; it uses less power to achieve the same hydrogen pumping rate as the ALD catalyst (Figure 9). The rate of hydrogen pumping is proportional to the electrical current drawn from the cell. Comparing the particle characteristics in Figure 6, HiSPEC 4000 had smaller more dispersed particles, leading to a higher metal surface area and possibly more accessible sites for effective hydrogen pumping. Furthermore, the average particle size of HiSPEC 4000 might facilitate hydrogen pumping more effectively. To further test the correlation between nanoparticle character and hydrogen pumping performance, we explored an alternative ALD chemistry (section 3.4).

\subsection{Refining ALD chemistry by using hydrogen as a second precursor}

To adjust the Pt particle characteristics, we altered the ALD chemistry by using hydrogen as a second precursor instead of oxygen. All other process conditions remained invariant. Both hydrogen and oxygen can react with and volatilize ligands on deposited platinum. However, hydrogen will be less reactive with the catalyst support. Furthermore, hydrogen dosing leaves no absorbed oxygen on the surface, making the 
surface more inert in subsequent ALD cycles [32]. Overall, the chemistry is most likely more gentle, allowing the generation of finer, more dispersed, Pt nanoparticles.

The effect of hydrogenation during ALD is shown in Figure 10. The resultant platinum particles were smaller and more dispersed than both the ALD catalyst synthesized using oxygen as a second precursor and the commercial equivalents. Notably the weight loading of the platinum was lower, most likely due to a lower deposition rate and preservation of the carbon support. Mass spectra from the FBR suggested a gentle chemistry consistent with refined nanoparticle creation (Figure 11). Part A of Figure 11 shows that combustion was not a significant contributor to ligand removal. This stands in sharp contrast to the chemistry seen when dosing oxygen (section 3.2). Although some $\mathrm{CO}_{2}$ was observed during platinum deposition, little was seen while dosing hydrogen as the second precursor. Instead an abundance of hydrocarbons was produced (Part B of Figure 11) indicating that platinum precursor ligands are lost via hydrogenation. This result has been observed in prior research [38]. Unexpectedly, the hydrogen signal increased only subtly during the hydrogen dose (Part A of Figure 11). Under the given conditions breakthrough might not have occurred. Furthermore, because less platinum was deposited using this hydrogenation chemistry, likely less precursor was utilized versus the combustion chemistry process.

\subsection{Hydrogen pumping with Pt catalysts from ALD using hydrogen as a second precursor}

The improved ALD catalyst and its commercial analog (Premetek P10A200) were integrated into electrodes and membrane electrode assemblies for hydrogen pump testing. The ALD catalyst appears to perform similarly to its commercial counterpart, Premetek P10A200 (Figure 12). Average voltages necessary to produce 8 amps of current for each catalyst are listed in Table 2, which shows the ALD catalyst made with hydrogen as a second precursor and the commercial catalysts require similar amounts of voltage for the equivalent amount of hydrogen pumping. These results are consistent with the improved nanoparticle character yielded by the revised ALD process (section 3.4). 


\subsection{Conclusions}

Platinum ALD catalysts were synthesized using two chemistries related to the removal of platinum precursor hydrocarbons. First, combustion was used to burn off residual platinum ligands. The resultant catalyst was composed of nanoparticles that were large and heterogeneous relative to a commercial counterpart (HiSPEC 4000). This ALD catalyst underperformed HiSPEC 4000 in hydrogen pumping tests. Second, hydrogenation was used to remove residual platinum ligands. During ALD, a less combustive chemistry was observed by in situ mass spectrometry. This improved process produced a catalyst composed of nanoparticles that were small and uniform relative to the other catalysts examined. Hydrogen pumping with this catalyst was similar to its commercial counterpart (Premetek P10A200), a result that illustrates the flexibility and potential of ALD - especially since the commercial catalysts have been optimized for performance. Given the scalable nature of a fluidized bed reactor, this particle ALD process shows considerable promise for producing high-performance Pt-based catalysts for hydrogen pumping.

\section{Acknowledgments}

This work was supported by the National Science Foundation Graduate Research Fellowship Program. The authors thank Fred Luizer at University of Colorado Boulder Laboratory of Environmental and Geological Studies (LEGS) for conducting ICP-OES analysis, and Troy Gould for conducting TEM analysis and for assistance with vacuum IR spectroscopy, Carolyn Schoenbaum for assistance in conducting DRIFTS analysis, and also thank Caitlin Majlinger, Chip Fisher, and Boris Chubukov at University of Colorado Boulder Department of Chemical and Biological Engineering for chemisorption and LECO analysis. Prof. Weimer acknowledges insightful discussions with Prof. Mooson Kwauk during a number of International Fluidization Conferences. 


\section{References}

1. Department of Energy, The Department of Energy Hydrogen and Fuel Cells Program Plan. US Department of Energy, Hydrogen and Fuel Cells Program, 2011.

2. Lu, G.Q., et al., Inorganic membranes for hydrogen production and purification: A critical review and perspective. Journal of Colloid and Interface Science, 2007. 314(2): p. 589-603.

3. Sedlak, J.M., J.F. Austin, and A.B. Laconti, HYDROGEN RECOVERY AND PURIFICATION USING THE SOLID POLYMER ELECTROLYTE ELECTROLYSIS CELL. International Journal of Hydrogen Energy, 1981. 6(1): p. 45-51.

4. $\quad$ Phair, J.W. and S.P.S. Badwal, Review of proton conductors for hydrogen separation. Ionics, 2006. 12(2): p. 103-115.

5. Eisman, G.A. and D.L. Ludlow, Integrated electrochemical hydrogen compression systems. 2007, H2 Pump Llc.

6. Nørskov, J.K., et al., Trends in the Exchange Current for Hydrogen Evolution. Journal of the Electrochemical Society, 2005. 152(3): p. J23-J26.

7. Steele, B.C.H. and A. Heinzel, Materials for fuel-cell technologies. Nature, 2001. 414(6861): p. 345352.

8. Esparbe, I., et al., Structure and electrocatalytic performance of carbon-supported platinum nanoparticles. Journal of Power Sources, 2009. 190(2): p. 201-209.

9. King, D.M., X. Liang, and A.W. Weimer, Functionalization of fine particles using atomic and molecular layer deposition. Powder Technology, 2012. 221(0): p. 13-25.

10. Detavernier, C., et al., Tailoring nanoporous materials by atomic layer deposition. Chemical Society Reviews, 2011. 40(11): p. 5242-5253.

11. van Ommen, J.R., et al., Scalable gas-phase processes to create nanostructured particles. Particuology, 2010. 8(6): p. 572-577.

12. Ferguson, J.D., A.W. Weimer, and S.M. George, Atomic layer deposition of ultrathin and conformal Al2O3 films on BN particles. Thin Solid Films, 2000. 371(1-2): p. 95-104.

13. Wank, J.R., S.M. George, and A.W. Weimer, Nanocoating individual cohesive boron nitride particles in a fluidized bed by ALD. Powder Technology, 2004. 142(1): p. 59-69.

14. Hakim, L.F., et al., Nanocoating Individual Silica Nanoparticles by Atomic Layer Deposition in a Fluidized Bed Reactor. Chemical Vapor Deposition, 2005. 11(10): p. 420-425.

15. Longrie, D., D. Deduytsche, and C. Detavernier, Reactor concepts for atomic layer deposition on agitated particles: A review. Journal of Vacuum Science \&amp; Technology A, 2014. 32(1): p. 010802.

16. Grillo, F., M.T. Kreutzer, and J.R. van Ommen, Modeling the precursor utilization in atomic layer deposition on nanostructured materials in fluidized bed reactors. Chemical Engineering Journal, 2015. 268: p. 384-398.

17. King, D.M., et al., Atomic layer deposition on particles using a fluidized bed reactor with in situ mass spectrometry. Surface \& Coatings Technology, 2007. 201(22-23): p. 9163-9171.

18. Goulas, A. and J.R. van Ommen, Scalable Production of Nanostructured Particles using Atomic Layer Deposition. KONA Powder and Particle Journal, 2014. 31: p. 234-246.

19. Liang, X. and A. Weimer, Photoactivity passivation of TiO2 nanoparticles using molecular layer deposited (MLD) polymer films. Journal of Nanoparticle Research, 2010. 12(1): p. 135-142.

20. Gould, T.D., et al., Synthesis of supported Ni catalysts by atomic layer deposition. Journal of Catalysis, 2013. 303(0): p. 9-15.

21. Marichy, C., M. Bechelany, and N. Pinna, Atomic Layer Deposition of Nanostructured Materials for Energy and Environmental Applications. Advanced Materials, 2012. 24(8): p. 1017-1032. 
22. Hsieh, C.T., et al., Atomic layer deposition of Pt nanocatalysts on graphene oxide nanosheets for electro-oxidation of formic acid. International Journal of Hydrogen Energy, 2012. 37(23): p. 1783717843.

23. King, J.S., et al., Ultralow loading Pt nanocatalysts prepared by atomic layer deposition on carbon aerogels. Nano Letters, 2008. 8(8): p. 2405-2409.

24. Liu, C., et al., Atomic Layer Deposition of Platinum Nanoparticles on Carbon Nanotubes for Application in Proton-Exchange Membrane Fuel Cells. Small, 2009. 5(13): p. 1535-1538.

25. Deng, S., et al., Synthesis of a 3D network of Pt nanowires by atomic layer deposition on a carbonaceous template. Nanoscale, 2014. 6(12): p. 6939-6944.

26. Bauer, E., Phänomenologische theorie der kristallabscheidung an oberflächen. II. Zeitschrift für Kristallographie-Crystalline Materials, 1958. 110(1-6): p. 395-431.

27. Marichy, C. and N. Pinna, Carbon-nanostructures coated/decorated by atomic layer deposition: Growth and applications. Coordination Chemistry Reviews, 2013. 257(23-24): p. 3232-3253.

28. Liang, X., et al., Reaction mechanism studies for platinum nanoparticle growth by atomic layer deposition. Journal of Nanoparticle Research, 2011. 13(9): p. 3781-3788.

29. Juda, W., R. Bombard, and C. Kneger, Electrochemical pressurizer/purifier of hydrogen for operation at moderately elevated temperatures (including high-temperature electrochemical pump in a membrane generator of hydrogen. 2003, Walter Juda, Bombard R. Todd, Kneger Charles W.

30. Liang, X., et al., Scalable synthesis of palladium nanoparticle catalysts by atomic layer deposition. Journal of Nanoparticle Research, 2012. 14(6): p. 1-12.

31. Lubers, A., et al., Mechanistic studies for depositing highly dispersed Pt nanoparticles on carbon by use of trimethyl(methylcyclopentadienyl)platinum(IV) reactions with $\mathrm{O} 2$ and $\mathrm{H} 2$. Journal of Nanoparticle Research, 2015. 17(4): p. 1-16.

32. George, S.M., Atomic Layer Deposition: An Overview. Chemical Reviews, 2010. 110(1): p. 111-131.

33. El-Hendawy, A.-N.A., Influence of $\mathrm{HNO} 3$ oxidation on the structure and adsorptive properties of corncob-based activated carbon. Carbon, 2003. 41(4): p. 713-722.

34. Mackus, A.J.M., et al., Influence of oxygen exposure on the nucleation of platinum atomic layer deposition: consequences for film growth, nanopatterning, and nanoparticle synthesis. Chemistry of Materials, 2013.

35. Mackus, A.J.M., et al., Catalytic Combustion and Dehydrogenation Reactions during Atomic Layer Deposition of Platinum. Chemistry of Materials, 2012. 24(10): p. 1752-1761.

36. Coblentz Society, I., Evaluated Infrared Reference Spectra, in NIST Chemistry WebBook, NIST Standard Reference Database Number 69, P.J. Linstrom and W.G. Mallard, Editors, National Institute of Standards and Technology: Gaithersburg MD.

37. Nguyen, M.T., et al., Characterisation of a electrochemical hydrogen pump using electrochemical impedance spectroscopy. Journal of Applied Electrochemistry, 2011. 41(9): p. 1033-1042.

38. Xue, Z., et al., Organometallic chemical vapor deposition of platinum. Reaction kinetics and vapor pressures of precursors. Chemistry of Materials, 1992. 4(1): p. 162-166. 


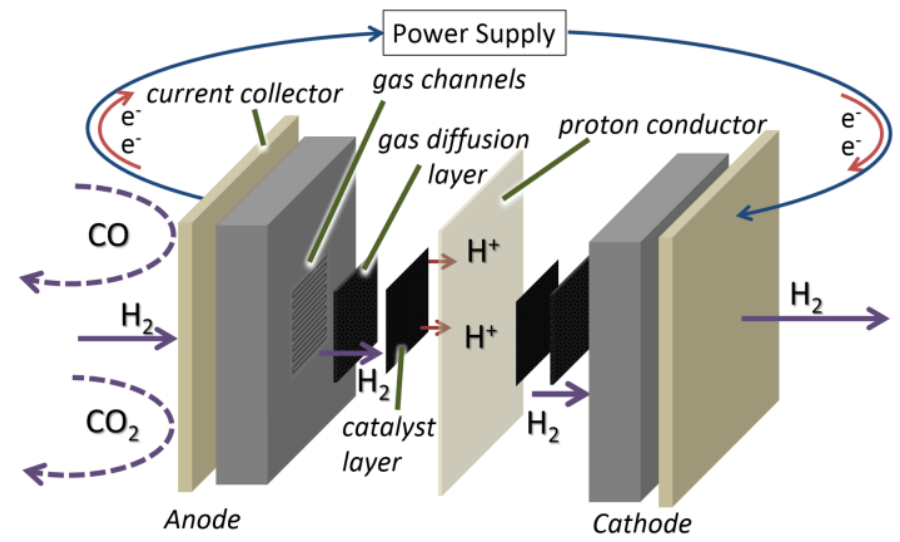

Figure 1. Schematic of a hydrogen pump using a proton conducting membrane.

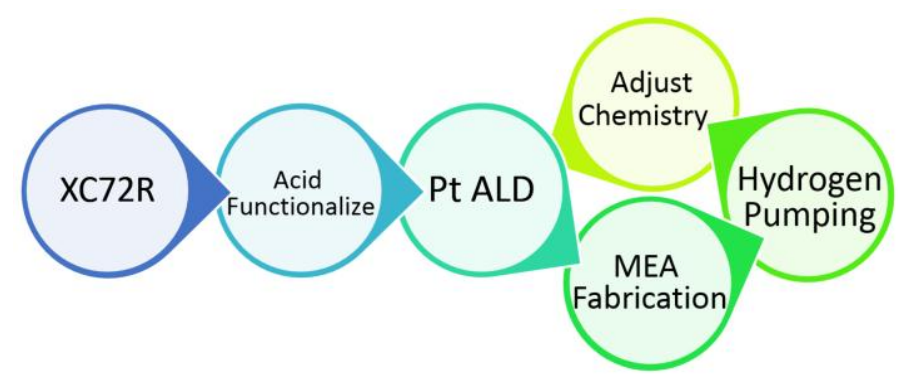

Figure 2. Process flow: from carbon to hydrogen pumping.

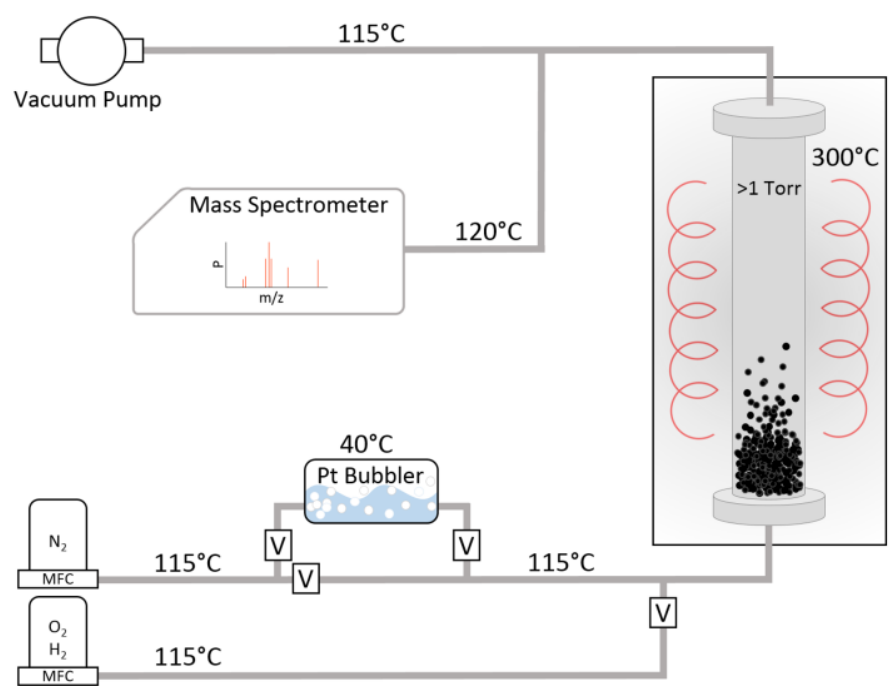

Figure 3. Schematic of a fluidized bed reactor ALD system. 


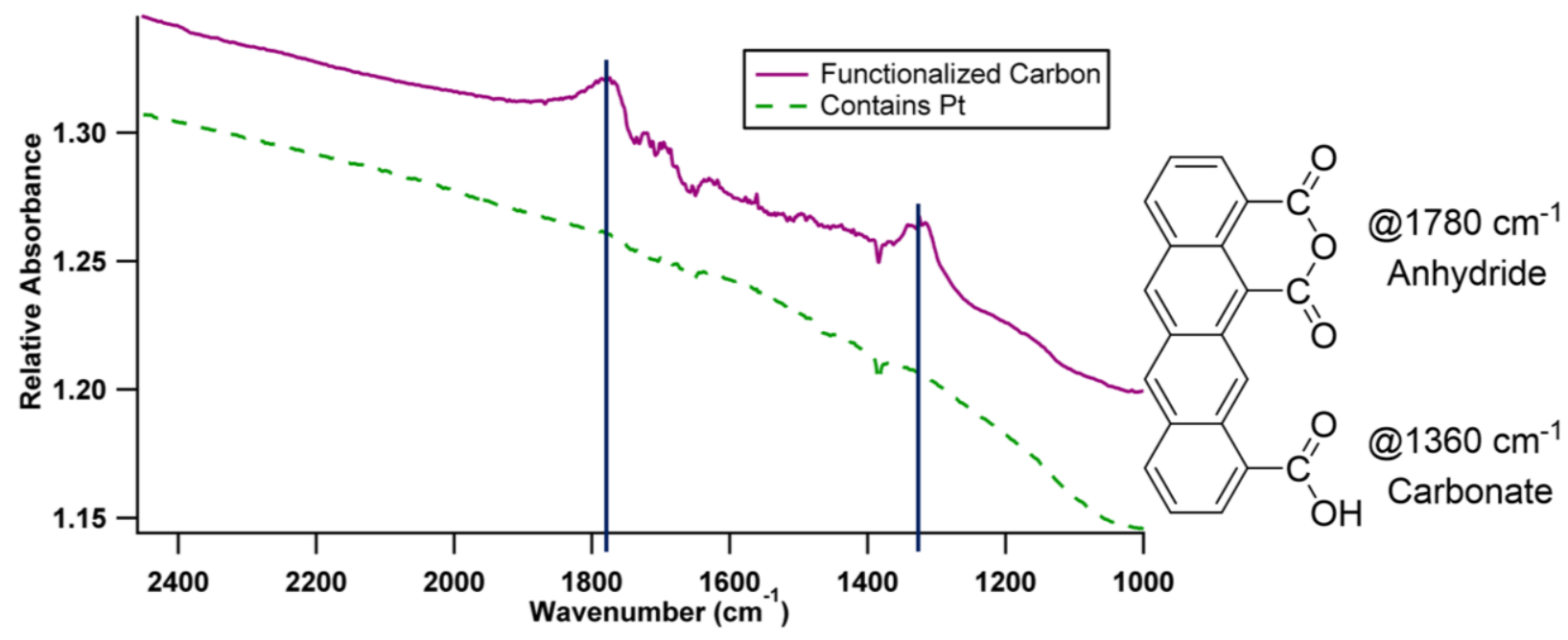

Figure 4. DRIFTS spectra of functionalized XC72R after platinum has been deposited via ALD. Both cases were normalized against unmodified XC72R. The peaks on the functionalized carbon correspond to anhydride and carbonate groups on the surface, which dissipate after platinum is deposited.

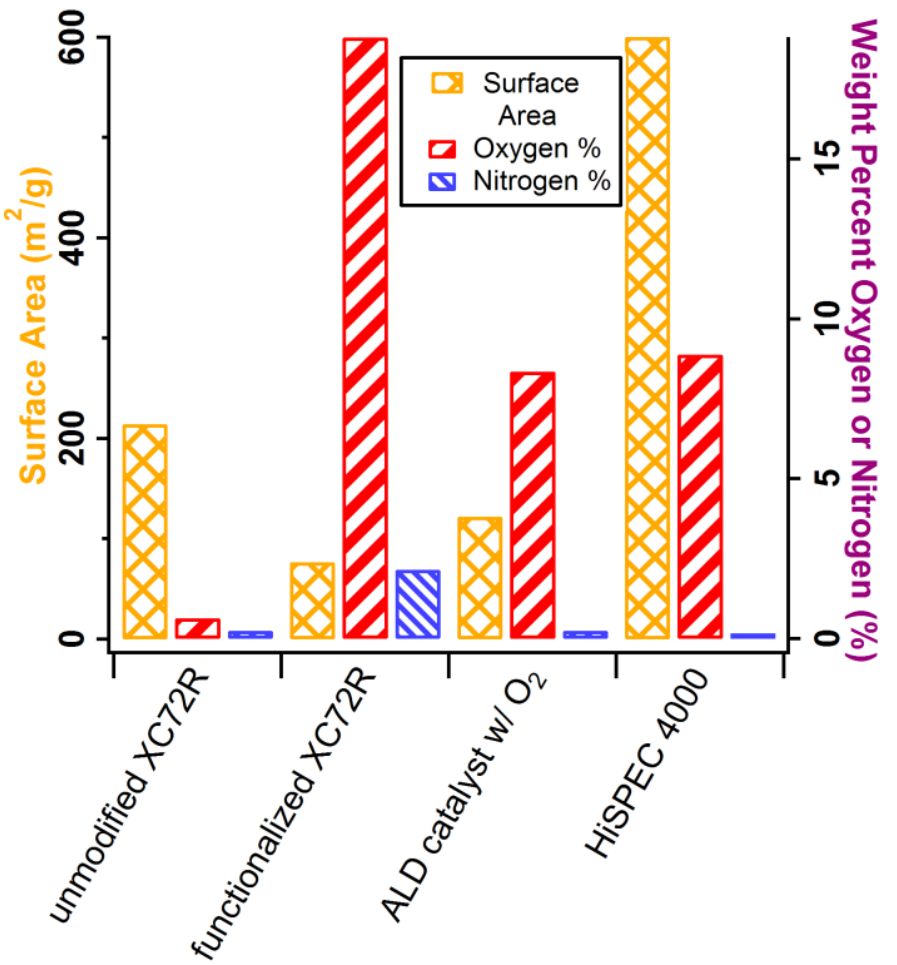

Figure 5. Summary of the surface area and composition of unmodified XC72R, functionalized XC72R, and platinum loaded XC72R with oxygen as a second precursor during ALD, and HiSPEC 4000. 


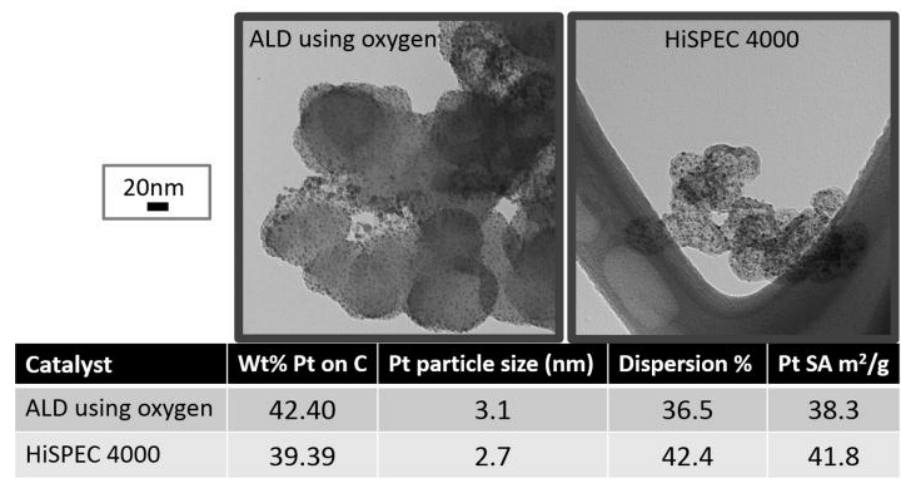

Figure 6. Transmission electron microscope imagery and platinum nanoparticle metrics of the Pt/C catalyst from ALD using oxygen and a commercial catalyst for comparison (HiSPEC 4000). In the images, platinum particles appear as black and carbon shows as grey.
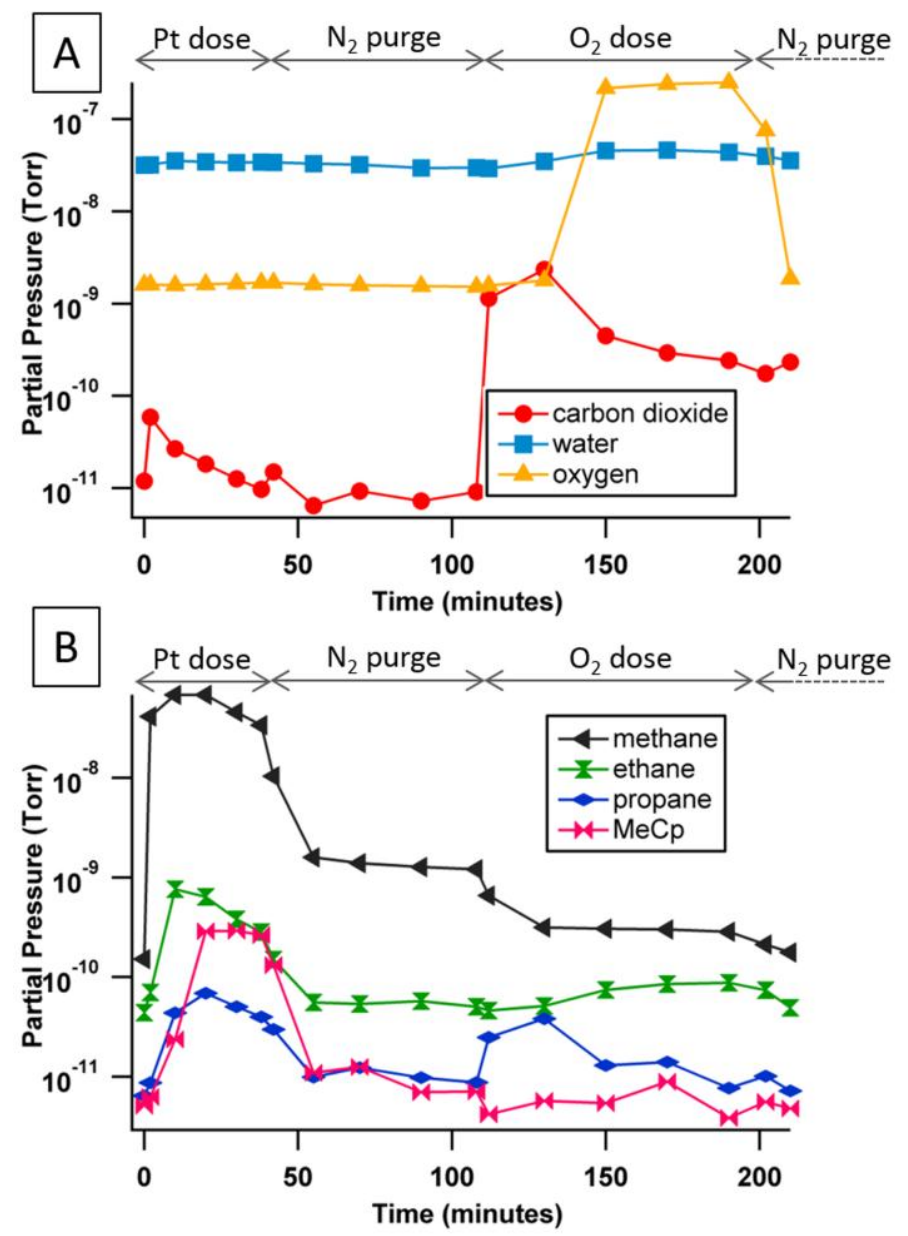

Figure 7. Mass spectra of FBR from the second ALD cycle using $\mathrm{O}_{2}$ as a second precursor. Part A: combustion species are tracked, including carbon dioxide, water and oxygen. Part B: hydrocarbon species are tracked, including methane, ethane, propane and methylcyclopentene (MeCp). 


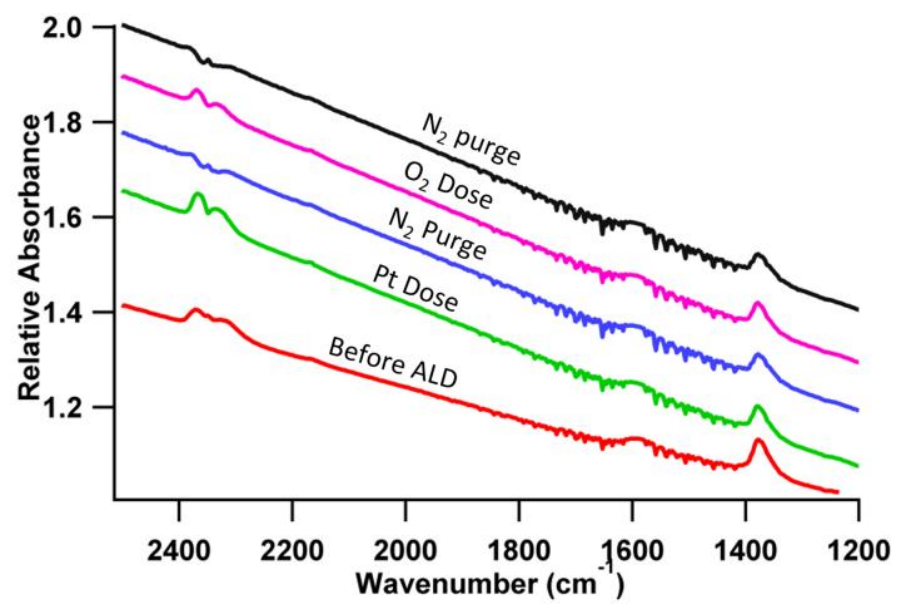

Figure 8. IR spectra of the second ALD cycle in a vacuum chamber.

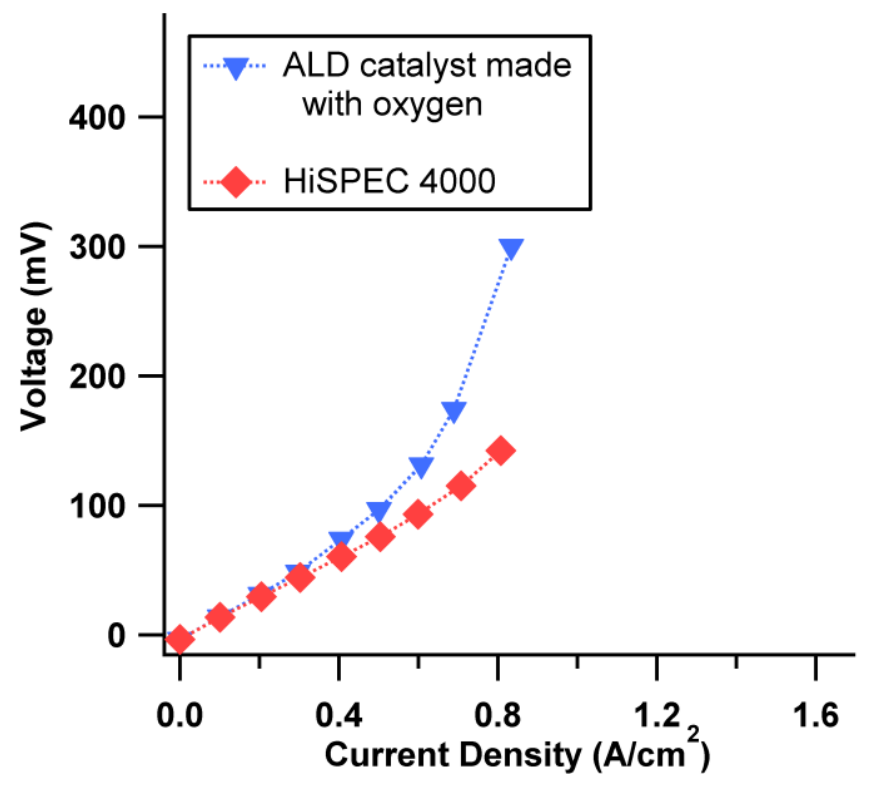

Figure 9. ALD catalyst made with oxygen as a second precursor and HiSPEC 4000 were evaluated as hydrogen pump catalysts. 


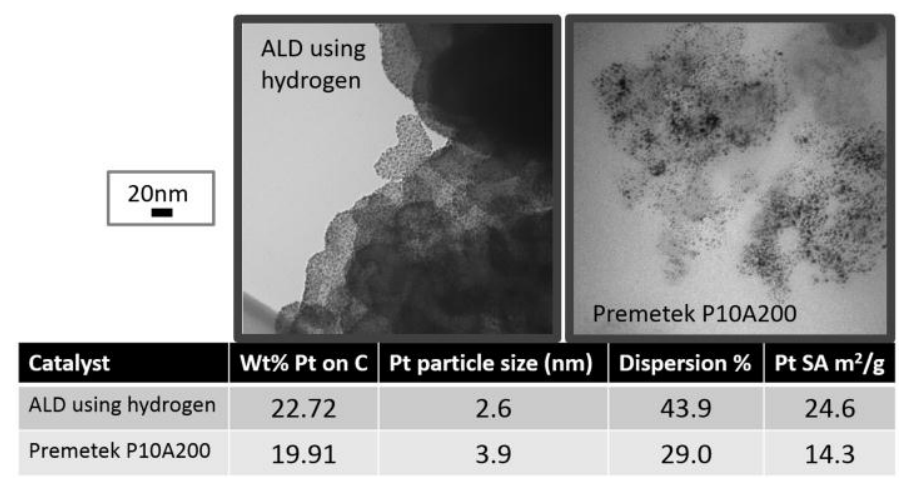

Figure 10. Transmission electron microscope imagery and platinum nanoparticle metrics of the Pt/C catalyst from ALD using hydrogen and its commercial analog (Premetek P10A200). In the images platinum particles appear as black and carbon shows as grey.

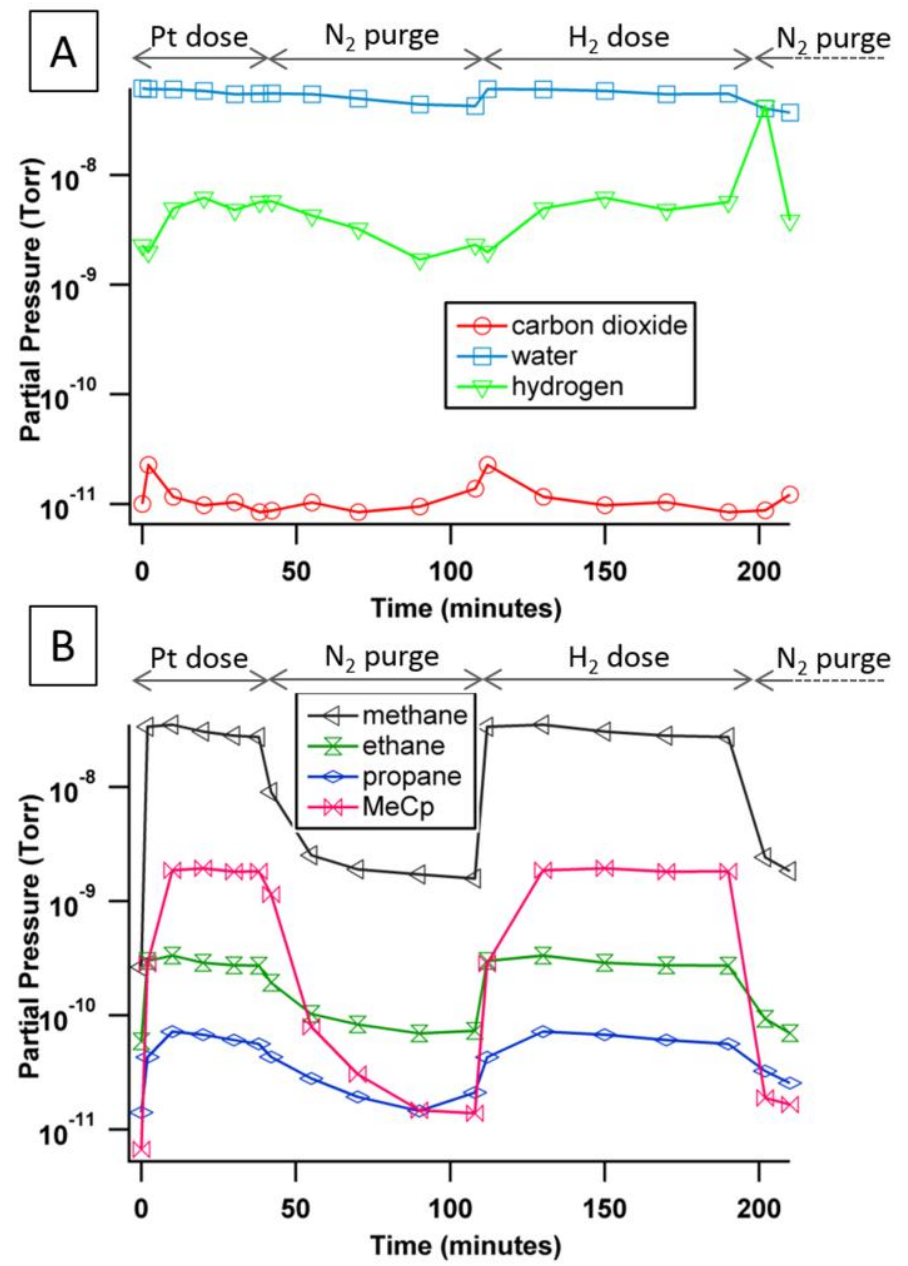

Figure 11. Mass spectra of reaction by-products during the second cycle of Pt ALD using hydrogen as a second precursor. Part A: combustion species are tracked, including carbon dioxide, and water, as well as hydrogen. Part B: hydrocarbon species are tracked, including methane, ethane, propane and methylcyclopentene (MeCp). 


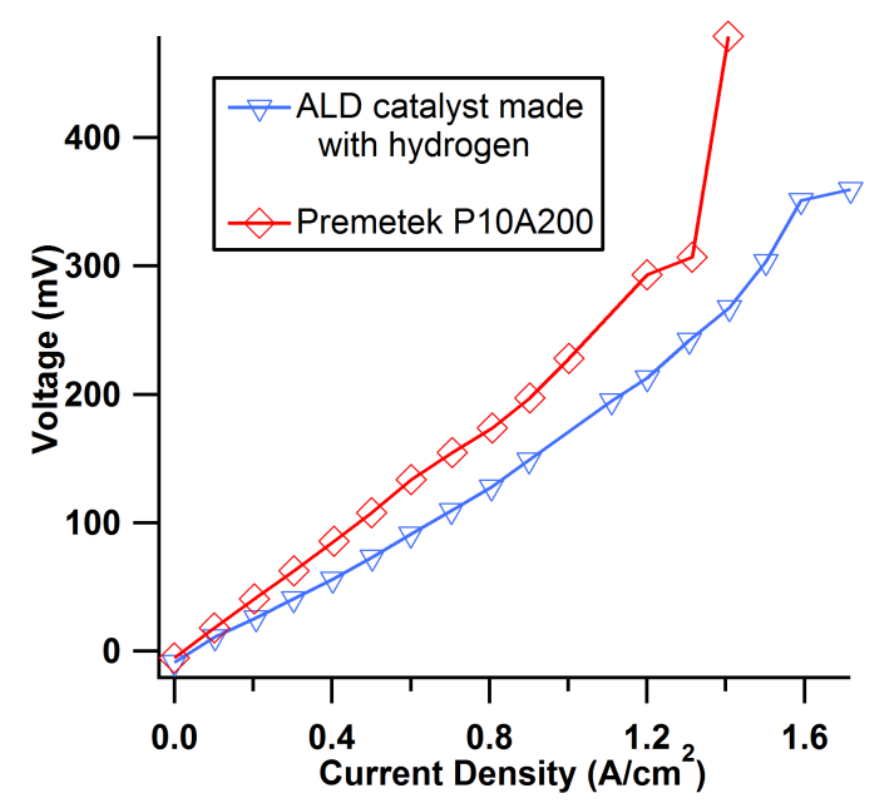

Figure 12. Comparing the ALD catalyst with hydrogen as a second precursor to the Premetek P10A200 commercial catalyst during hydrogen pumping.

Table 1. Membrane electrode assembly details.

\section{Anode Catalyst Cathode Catalyst Pt Weight Loading $\left(\mathrm{mg} / \mathrm{cm}^{2}\right)$}

\begin{tabular}{c|cc|}
\hline ALD via oxygen & ALD via oxygen & 0.15 \\
ALD via hydrogen & Premetek P10A200 & 0.3 \\
HiSPEC 4000 & HiSPEC 4000 & 0.15 \\
Premetek P10A200 & Premetek P10A200 & 0.3 \\
\cline { 2 - 3 } & &
\end{tabular}

Table 2. Required voltage for 8 amps of hydrogen pumping for each catalyst.

\section{Catalyst $\quad$ Voltage required for $8 \mathrm{~A}$ (in $\mathrm{mV}$ )}

\begin{tabular}{c|c|}
\hline ALD via oxygen & 300 \\
ALD via hydrogen & 127 \\
HiSPEC 4000 & 142 \\
Premetek P10A200 & 137 \\
\hline
\end{tabular}




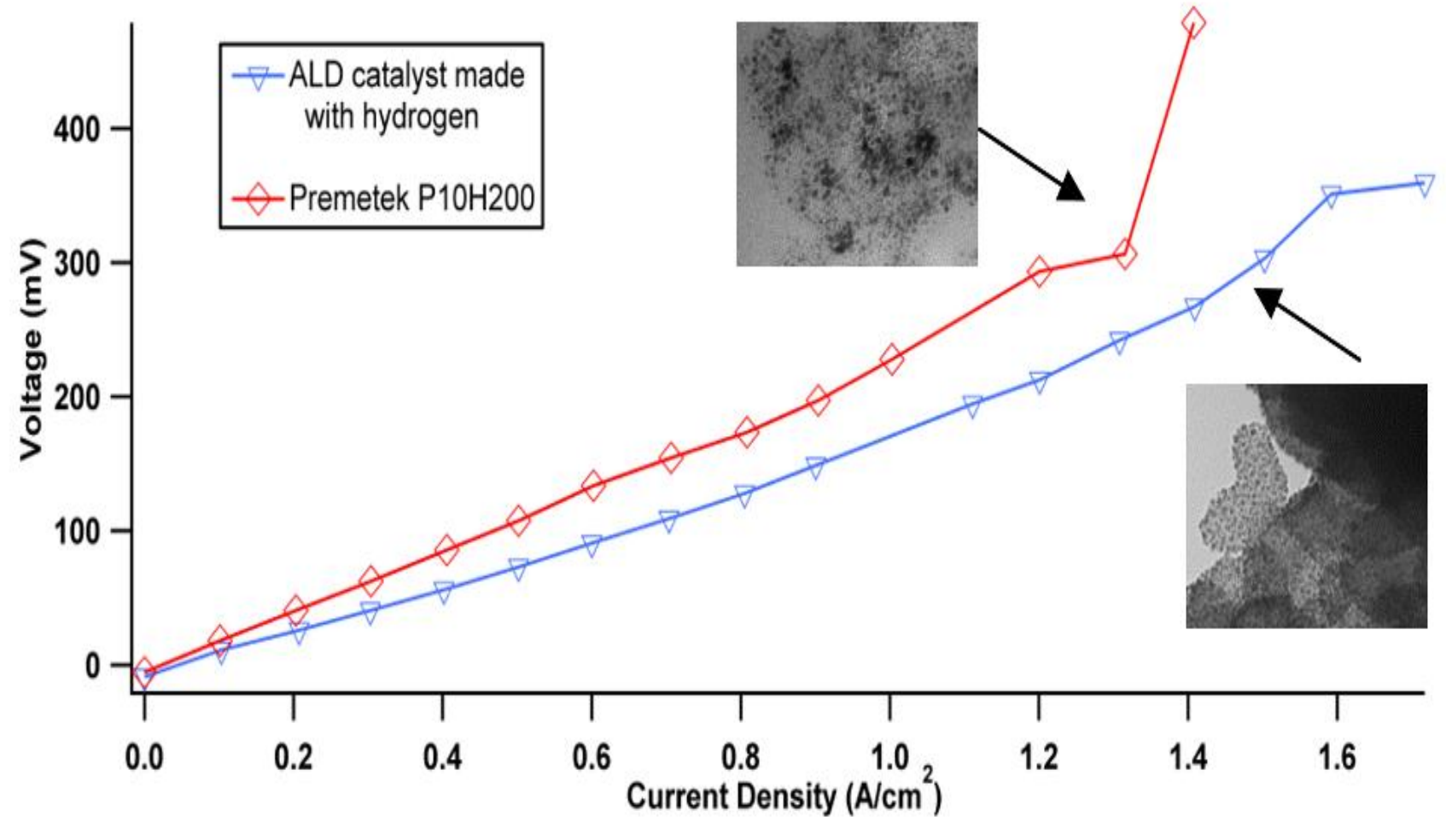

